LESSON OF THE MONTH

\title{
Pulmonary arterial hypertension in systemic sclerosis: diagnostic pathway and therapeutic approach
} L Robertson, A Pignone, O Kowal-Bielecka, G Fiori, C P Denton, M Matucci-Cerinic

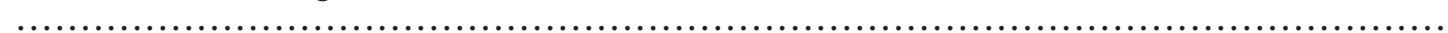

Ann Rheum Dis 2005;64:804-807. doi: 10.1136/ard.2004.026427

\section{CASE HISTORIES \\ Case 1}

A 63 year old white woman was admitted to hospital in February 2000 owing to rapidly increasing dyspnoea on exertion (NYHA III), chest pain, and fever. The presence of sclerodactyly with loss of distal parts of the fingers, and megacapillaries at capillaroscopy, allowed the diagnosis of limited cutaneous systemic sclerosis (fig 1). Physical examination also disclosed crackles in the lower lobes of the lungs, systolic murmur with loud pulmonary second sound, and feet oedema.

Laboratory tests included high erythrocyte sedimentation rate $(145 \mathrm{~mm} / \mathrm{lst} \mathrm{h}), \mathrm{C}$ reactive protein $(2530 \mathrm{mg} / \mathrm{l}$, normal $<100 \mathrm{mg} / \mathrm{l})$, presence of antinuclear antibodies (1/1280 and speckled pattern on indirect immunofluorescence), mild anaemia and thrombocytopenia (platelet count of $\left.115 \times 10^{9} / 1\right)$, decreased arterial oxygen and carbon dioxide pressures. A chest $x$ ray examination showed cardiomegaly, dilatation of the main pulmonary artery (fig l), centralisation of pulmonary vascular flow, and reticular opacities in the lower parts of the lungs. Electrocardiography (ECG) at admission did not show any significant abnormalities. Doppler echocardiography disclosed a pulmonary artery systolic pressure (PASP) of $86 \mathrm{~mm} \mathrm{Hg}$, suggesting a severe pulmonary artery hypertension (PAH). Pulmonary function tests showed a reduction of forced vital capacity (FVC, $45 \%$ of predicted), and a high resolution chest computed tomography (CT) scan showed interstitial lung disease (ground glass and interstitial fibrosis) (fig 1). Furthermore, the patient developed pulmonary embolism, confirmed with perfusion scintigraphy (fig 1), with an increase in pulmonary pressure up to $115 \mathrm{~mm} \mathrm{Hg}$. Anticardiolipin antibodies were found to be positive.

The patient was treated with warfarin (international normalisation ratio (INR) between 2 and 3) and a high dose of calcium channel blocker, (nifedipine $120 \mathrm{mg} / \mathrm{day}$ ), an angiotensin converting enzyme (ACE) inhibitor (perindopril $4 \mathrm{mg} /$ day), diuretics (furosemide $20 \mathrm{mg} /$ day, spironolactone $100 \mathrm{mg} /$ day), and oxygen (1-2 litres/min). Owing to interstitial lung disease, confirmed at bronchoalveolar lavage (no infection detected, total cell number $200 \times 10^{9} / \mathrm{l}$, neutrophils $4 \%$, eosinophils $0 \%$, lymphocytes $23 \%$, macrophages $73 \%$ ), she also received cyclophosphamide (CYC) pulses $\left(1.0 \mathrm{~g} / \mathrm{m}^{2} /\right.$ month for 6 months) and prednisone $15 \mathrm{mg} /$ day. The treatment led to a gradual improvement in the patient's clinical status, lung function tests (FVC 80\%) and decrease in PASP (78 mm Hg). Within the next 6 months CYC infusions were repeated twice and then discontinued.

The patient continues to receive oral anticoagulants, a high dose calcium channel blocker, diuretics (furosemide $20 \mathrm{mg}$ every second day, spironolactone $25 \mathrm{mg} /$ day), an ACE inhibitor, prednisone $(5 \mathrm{mg} / \mathrm{day})$, and oxygen treatment. She feels well, reporting only sporadic oedema of the legs in the evening and dyspnoea on exertion (NYHA II). Her PASP in June 2003 was $68 \mathrm{~mm} \mathrm{Hg}$.

\section{Case 2}

In April 2002 a 69 year old white woman with diffuse systemic sclerosis (SSc), diagnosed in 2001, was admitted to hospital after a 6 month history of dyspnoea on exertion (NYHA II/III), fatigue, chest pain, and dry cough. Physical examination showed diffuse teleangiectasias, cyanosis of the lips and fingers, mild oedema of the feet, crackles in lower lobes, pansystolic murmur (4/6 L) on the apex, with accentuation of the pulmonary component of the second heart sound; the liver was enlarged with mild ascites.

Laboratory testing showed an erythrocyte sedimentation rate of $31 \mathrm{~mm} / \mathrm{lst} \mathrm{h}$; prothrombin time 20 seconds (normal $<15$ seconds), and activated partial prothrombin time 41 seconds ( normal 26-36 seconds). Antinuclear antibodies were positive (1/5120). A chest $x$ ray showed cardiomegaly without interstitial involvement. FVC was normal (84\%), with a significant decrease of carbon monoxide transfer factor TLCO $(36 \%)$, severe hypoxaemia and respiratory alkalosis (partial pressure of oxygen $62.6 \mathrm{~mm} \mathrm{Hg}$; partial pressure of carbon dioxide $28 \mathrm{~mm} \mathrm{Hg} ; \mathrm{HCO}_{3}{ }^{-} 20.7 \mathrm{mmol} / \mathrm{l}$; arterial oxygen saturation $90 \%$ ). Chest high resolution CT showed an enlarged right atrium and ventricle, dilatation of the main pulmonary artery, centralisation of pulmonary vascular flow with pulmonary congestion, bilateral pleural effusion, and pericardial effusion. Bibasilar mild ground glass opacities were detected. A Doppler echocardiogram showed enlargement of the right heart with tricuspid insufficiency, paradoxical movement of the cardiac septum, mild pericardial effusion, and raised PASP (70 $\mathrm{mm} \mathrm{Hg}$ ).

Right cardiac catheterisation confirmed severe PAH with a positive test for resistance responders: mean right atrial pressure $=20 \mathrm{~mm} \mathrm{Hg}$ at rest and $19 \mathrm{~mm} \mathrm{Hg}$ after the epoprostenol test, PASP $56 \mathrm{~mm} \mathrm{Hg}$ at rest and $50 \mathrm{~mm} \mathrm{Hg}$ after the epoprostenol test, mean pulmonary capillary wedge pressure $13 \mathrm{~mm} \mathrm{Hg}$, reduced cardiac output of 2.50 litres/min that became 4.40 litres/min after the epoprostenol test, and pulmonary vascular resistance of $22.4 \mathrm{U} / \mathrm{W}$ at rest and $11.3 \mathrm{U} / \mathrm{W}$ after the epoprostenol test.

\section{DISCUSSION}

\section{Remarks on case 1}

Key questions

- Are any further investigations necessary?

- Is it appropriate to initiate anticoagulation?

- Was $\mathrm{O}_{2}$ necessary?

\footnotetext{
Abbreviations: $\mathrm{ACE}$, angiotensin converting enzyme; $\mathrm{CYC}$, cyclophosphamide; ECG, electrocardiography; FVC, forced vital capacity; PAH, pulmonary artery hypertension; PASP, pulmonary artery systolic pressure; SSC, systemic sclerosis
} 

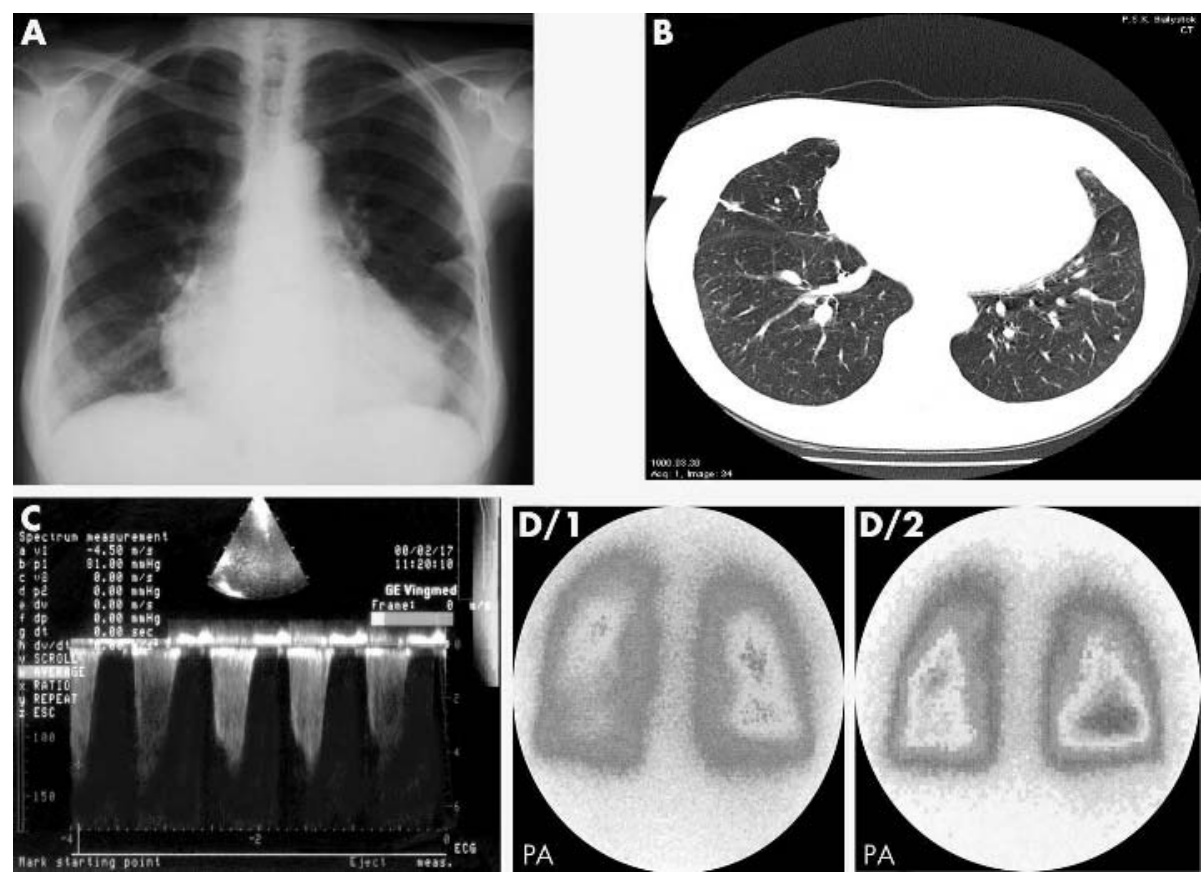

Figure 1 Case 1. (A) Chest $x$ ray findings show bibasilar reticular opacities, cardiomegaly with accentuation of the right ventricle, and enlargement of the pulmonary artery, which are consistent with interstitial lung disease and pulmonary hypertension. (B) High resolution CT of the lungs discloses features of interstitial lung disease with "ground glass" opacities and widening of the central blood vessels. (C) Doppler echocardiography shows blood regurgitation through the tricuspid valve, which allows estimation of PASP. (D) Perfusion scintigraphy of the lungs carried out in February 2000 $(D / 1)$ shows redistribution of the blood flow into the upper parts of the lungs and irregular areas of diminished blood flow in the peripheral parts of the lungs consistent with PAH and pulmonary thromboembolism. For comparison, perfusion scintigraphy of the lungs carried out in June 2000 (D/2) shows a significant increase in blood flow in the lower parts of both lungs, which paralleled a decrease in PASP as measured with Doppler echocardiography.

Facts in this case

- Pulmonary function tests indicated a "restrictive pattern" with a reduction of the FVC.

- A chest CT scan indicated interstitial fibrosis and alveolitis.

- Doppler echocardiography disclosed severe pulmonary hypertension.

- The immune profile identified anticardiolipin antibodies that together with thrombocytopenia and pulmonary thromboembolism allowed the diagnosis of antiphospholipid syndrome.

- ECG did not show any modifications. In fact, ECG may be an inadequate tool for ruling out the presence of pulmonary hypertension, in particular in early phases.

- A standard chest $x$ ray showed typical signs of PAH such as "dilated pulmonary arteries" and "increased cardiothoracic ratio".

- This is a case of limited cutaneous SSc. In such a case, when the patient complains of breathlessness, interstitial involvement or vascular lung disease, or both, should be suspected, both leading to PAH and respiratory failure. ${ }^{1}$ In this case, PAH was secondary to interstitial lung disease, as indicated by a reduced FCV and the CT scan and was exacerbated by a pulmonary thromboembolic event due to antiphospholipid syndrome. The relationship between PAH and interstitial disease is confirmed by the results of the treatment with CYC, which significantly improved the FVC and PASP.

\section{Comment}

This case shows that some important investigations, that might have added significant information for a more precise management, are missing:
- Carbon monoxide transfer factor (TLCO) is missing. It measures gas exchange at the level of the alveolocapillary membrane and identifies impairment of the transfer of CO that may be due to interstitial fibrosis or vascular disease. In particular, the FVC/TLCO ratio may be helpful in a differential diagnosis for PAH complicating SSc, with an FVC/TLCo ratio $>1.4$ indicating a vascular pulmonary hypertension. Accordingly, an isolated decrease of TLCo, with a normal FVC, is a reliable marker for the development of PAH in SSc. ${ }^{1-3}$ In SSc, a severe reduction in TLCO $(<40 \%$ of predicted values) is indeed an adverse prognostic factor. ${ }^{4}$ For this reason, pulmonary function tests with TLCO are a fundamental investigation in assessment of the patient with SSc. ${ }^{5}$

- Right heart catheterisation with vasodilator test. When the clinical assessment and the echo Doppler measurement indicate PAH then right heart catheterisation is mandatory. It allows the "mean pulmonary artery pressure" to be determined. Also, assessment of other indices such as right atrial and ventricular pressures, pulmonary capillary wedge pressure, and cardiac output is essential for differential diagnosis of PAH secondary to myocardial disease and has important prognostic value. Catheterisation makes it possible to verify if $\mathrm{PAH}$ is postcapillary, which is seldom the case in patients with SSc. A vasodilator test is necessary to evaluate the response of pulmonary vasculature in order to identify those patients who may benefit from the use of calcium channel blocker or other drugs, ${ }^{36}$ although the frequency of true responders is low in scleroderma and the benefits of high dose calcium channel blockers must be balanced against potential toxicity.

- Helical spiral CT angiography is indicated for further diagnosis and localisation of thromboembolic disease, 


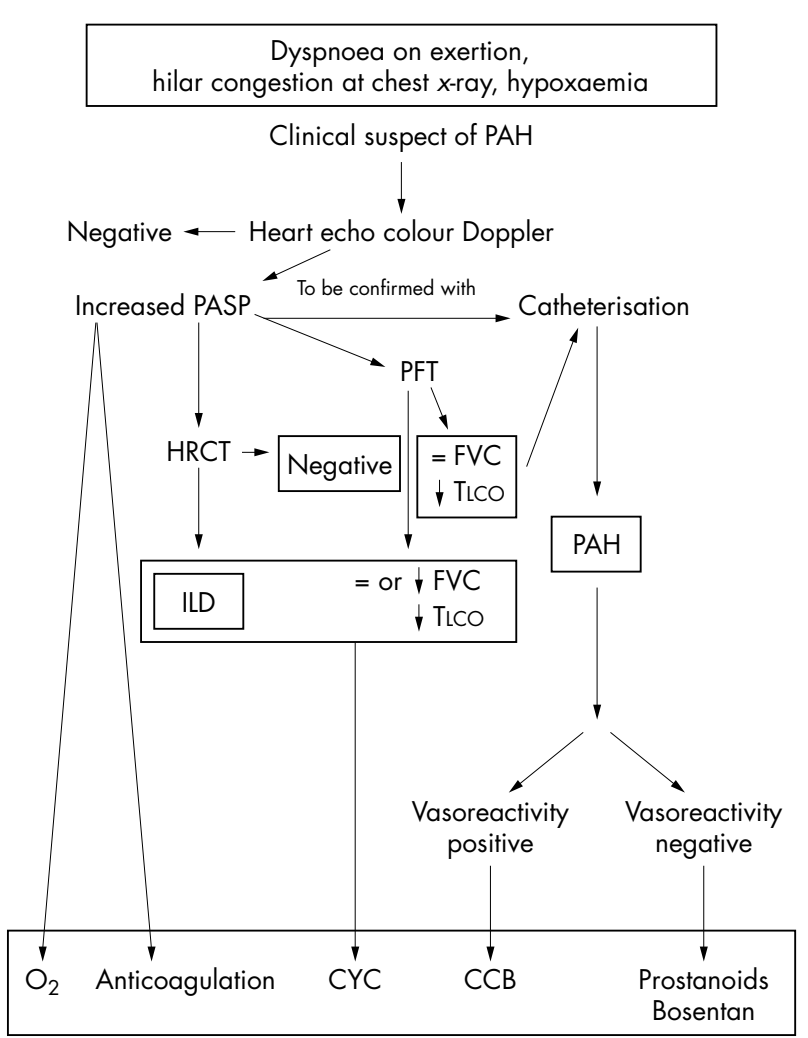

Figure 2 The flow chart suggests the diagnostic procedure and the possible therapeutic options for PAH in SSc. PASP, pulmonary artery systolic pressure; PFT, pulmonary function tests; TLCO, carbon monoxide transfer factor; FVC, forced vital capacity; CCB, calcium channel blockers; ILD, interstitial lung disease; $\mathrm{HRCT}$, high resolution computed tomography; CYC, cyclophosphamide; PAH, pulmonary arterial hypertension.

especially when surgical intervention is considered for embolectomy. ${ }^{7}$

- An exercise test (6 minute walk test) is recommended in patients with PAH at the time of diagnosis and for the follow up, to characterise the functional impairment and the response to treatment. ${ }^{36}$

The case was well managed, but

- Anticoagulation should be recommended in all cases of thromboembolic PAH and in all other types of arterial PAH unless contraindicated ${ }^{5}$

- Oxygen treatment is indeed recommended in all cases of PAH with hypoxaemia. ${ }^{5}$

\section{Remarks on case 2}

Key questions

- Are any further investigations necessary?

- Which is the correct therapeutic approach to this case?

Facts in this case

- The reduction of TLCO with a normal FVC indicates the possible involvement of the lung vasculature, leading to PAH.

- Doppler echocardiography confirms the suspicion of severe PAH.

- Catheterisation confirms the presence of PAH. Vasoreactivity indicates that there is a significant vasospastic component in this patient. Improved cardiac output after epoprostenol challenge suggests that long term vasodilators may be useful.

- Lung involvement is mild but is likely to be a cofactor, determining the degree of dyspnoea and functional impairment even though the prevalent involvement is of the lung vasculature

\section{Comment}

In this case, some investigations are still missing:

- Anticardiolipin antibodies, $\beta_{2}$-glycoprotein $I$, and lupus anticoagulant were not determined. Note that in this case the activated partial prothrombin time is prolonged: this may indicate an underlying antiphospholipid syndrome, as in case 1.

- Perfusion scintigraphy of the lungs/helical CT angiography is mandatory to exclude thromboembolic disease.

- An exercise test (6 minute walk test) may be helpful as in case 1.

In this case, basal therapy is needed: first of all it is necessary to anticoagulate (reach an INR of between 2 and 3), use oxygen (2 litres/min) that may be highly helpful, as well as diuretics. If during catheterisation the patient shows a positive response to vasodilatation, high dose calcium channel blockers may be used. If these fail to lower PAH, in patients in NYHA class III, bosentan or prostanoids may be used; in NYHA class IV, epoprostenol alone or in combination with bosentan may then be employed. When all these treatments fail to control PAH, atrioseptostomy or, eventually, heart-lung transplantation may be considered. ${ }^{3}{ }^{6}$

\section{THE LESSONS}

- In SSc, PAH is a severe complication that may rapidly lead to the patient's death; the early identification of PAH is mandatory to design a management plan to reduce pulmonary pressure (see fig 2 ).

- Cardiac Doppler echocardiography should be used regularly, including in asymptomatic patients; if a rise in pulmonary pressure is detected or, despite a negative Doppler echocardiography, a strong clinical suspicion for PAH exists, right catheterisation with vasoreactivity tests is mandatory to confirm PAH and to design the treatment.

- In cases of non-responsiveness to pharmacological vasoreactive treatments, a surgical approach (atrioseptotomy) should be considered. ${ }^{9}$

- In the presence of alveolitis, CYC treatment ${ }^{10-12}$ may be useful in order to control interstitial fibrosis (see fig 2), which contributes to the genesis of PAH.

\section{Authors' affiliations}

L Robertson, Rheumatology Unit, Royal Cornwall Hospital, Truro, Cornwall, UK

A Pignone, G Fiori, M Matucci-Cerinic, Department of Medicine, Division of Rheumatology, University of Florence, Florence, Italy O Kowal-Bielecka, Department of Rheumatology and Internal Diseases, Medical University of Bialystok, Bialystok, Poland

C P Denton, Division of Rheumatology, Royal Free Hospital, London, UK Series editor: Anthony D Woolf

Correspondence to: Professor M Matucci-Cerinic, Department of Medicine, Section of Rheumatology, Villa Monna Tessa, Viale Pieraccini 18, 50122 Firenze, Italy; cerinic@unifi.it

Accepted 22 December 2004 


\section{REFERENCES}

1 Steen VD, Graham G, Conte C, Owens G, Medsger TA Jr. Isolated diffusing capacity reduction in systemic sclerosis. Arthritis Rheum 1992;35:765-70.

2 Steen V, Medsger TA. Predictors of isolated pulmonary hypertension in patients with systemic sclerosis and limited cutaneous involvement. Arthritis Rheum 2003;48:516-22

3 Denton CP, Black CM. Pulmonary hypertension in systemic sclerosis. Rheum Dis Clin North Am 2003;29:335-50.

4 Peters-Golden M, Wise RA, Hochberg MC, Stevens MB, Wigley FM. Clinical and demographic predictors of loss of pulmonary function in systemic sclerosis. Medicine (Baltimore) 1984;63:221-3.

5 Matucci Cerinic M, D'Angelo S, Denton C, Vlachoyannopoulos P, Silver R. Assessment of lung involvement. Clin Exp Rheumatol 2003;21(suppl 29):s19-s24.

6 British Cardiac Society Guidelines and Medical Practice Committee, and approved by the British Thoracic Society and the British Society of Rheumatology. Recommendations on the management of pulmonary hypertension in clinical practice. Heart 2001;86(suppl 1):i1-13.
7 Bergin CJ, Sirlin CB, Hauschildt JP, Huynh TV, Auger WR, Fedullo PF, et al. Chronic thromboembolism: diagnosis with helical CT and MR imaging with angiographic and surgical correlation. Radiology 1997;204:695-702.

8 Fuster V, Steele PM, Edwards WD, Gersh BJ, McGoon MD, Frye RL. Primary pulmonary hypertension: natural history and the importance of thrombosis. Circulation 1984;70:580-7.

9 Galiè N, Seeger W, Naeije R, Simonneau G, Rubin L. Comparative analysis of clinical trials and evidence-based treatment algorithm in pulmonary arterial hypertension. J Am Coll Cardiol 2004:43:81-8s.

10 White B, Moore WC, Wigley FM, Xiao HQ, Wise RA. Cyclophosphamide is associated with pulmonary function and survival benefit in patients with scleroderma and alveolitis. Ann Intern Med 2000;132:947-54.

11 Giacomelli R, Valentini G, Salsano F, Cipriani P, Sambo P, Conforti ML, et al. Cyclophosphamide pulse regimen for the treatment of alveolitis in systemic sclerosis. J Rheumatol 2002;29:731-6.

12 Airò P, Danieli E, Parrinello G, Antonioli CM, Cavazzana I, Toniati P, et al. Intravenous cyclophosphamide therapy for systemic sclerosis. A single center experience and a review of the literature with pooled analysis of lung function tests results. Clin Exp Rheumatol 2004;22:573-8. 